\title{
Associated pathogenesis of bladder cancer and SARS-CoV-2 infection: a treatment strategy
}

\author{
Mukul Kumar Singh ${ }^{1} \cdot$ Mayank Jain $^{2} \cdot$ Hari Shyam $^{2} \cdot$ Pratap Shankar $^{2} \cdot$ \\ Vishwajeet $\operatorname{Singh}^{1}$ (B)
}

Received: 8 July 2021 / Accepted: 8 September 2021/Published online: 24 September 2021

(C) Indian Virological Society 2021

\begin{abstract}
Coronavirus disease 19 (Covid-19) is a pandemic that affects every human on Earth. Mortality rates are greater in people with both cancer and Covid-19. In comparison, patients with non-Muscle-Invasive Bladder Cancer (NMIBC) had reduced susceptibility to moderate Covid-19 mortality. The treatment and clinical management of NMIBC are consistent with BCG-mediated intravesical adjuvant therapy as a protective function against tumors. BCG vaccination exhibits a non-specific protective role against respiratory infections. This nonspecific effect of BCG is partially mediated by innate immune memory due to epigenetic changes in innate and adaptive immune system cells induced by the microbe. This editorial suggests that regularly test repurposed drugs include anti-cancer drugs till the proper antiviral drugs or vaccines will be developed.
\end{abstract}

Keywords Covid-19 · BCG $\cdot$ Bladder cancer

Mukul Kumar Singh, Mayank Jain and Vishwajeet Singh have contributed equally.

Agreement related to the accuracy or integrity of any part of the work are appropriately investigated and resolved; Agreement to be accountable for all aspects of the work.

Vishwajeet Singh

drvishwajeet68@gmail.com

1 Department of Urology, King George's Medical University, Lucknow 226003, India

2 Department of Centre For Advance Research, King George's Medical University, Lucknow 226003, India

\section{Dear editor}

The outbreak of SARS-CoV-2 become a pandemic in a very short period of time and the disease is caused by this virus is known as coronavirus disease 19 (Covid-19). This virus also has long-term ramifications as the pandemic progresses. The Angiotensin-converting enzyme 2 (ACE2) has the ability to dysregulate the immune system by virally mediating entry into epithelial cells of the respiratory system. Clinically, the outcome lead to respiratory distress syndrome with elevated inflammatory and anti-inflammatory markers. Although the compromised immune system coupled with the upregulation of inflammatory cytokines is a co-existing condition with cancer, patients with cancer and Covid-19 reported $28 \%$ mortality with a fatality rate of $37 \%$ due to induced multi-organ failure [1]. However, the severity and mortality of two disease condition potentially develop due to aging, sex, obesity, diabetes mellitus, smoking, and other comorbidities. Dai et al.; reported number of incidences and mortality rates among the standard metastatic cancer. In addition, the data revealed that patients with urothelial cancer had decreased exposure with moderate mortality rate against the Covid-19 infection [2]. This editorial therefore, elaborates on the shielding effect of Non-Muscle-Invasive Bladder Cancer (NMIBC) by decreasing the incidence and mortality of Covid-19 compared to common cancer.

Emerging evidence shows that bladder cancer entities with a high risk of NMIBC have an effect on the immune system and co-relate with Covid-19 infection by upregulation of inflammatory and cytokines (IL-6,2,8,10, TNF) production and by ensuing thrombo-inflammatory syndrome that increases the risk of mortality. In addition, the modified expression of neutrophils to lymphocytes (NLR) and platelet to lymphocytes ratio (PLR) is considered to be 

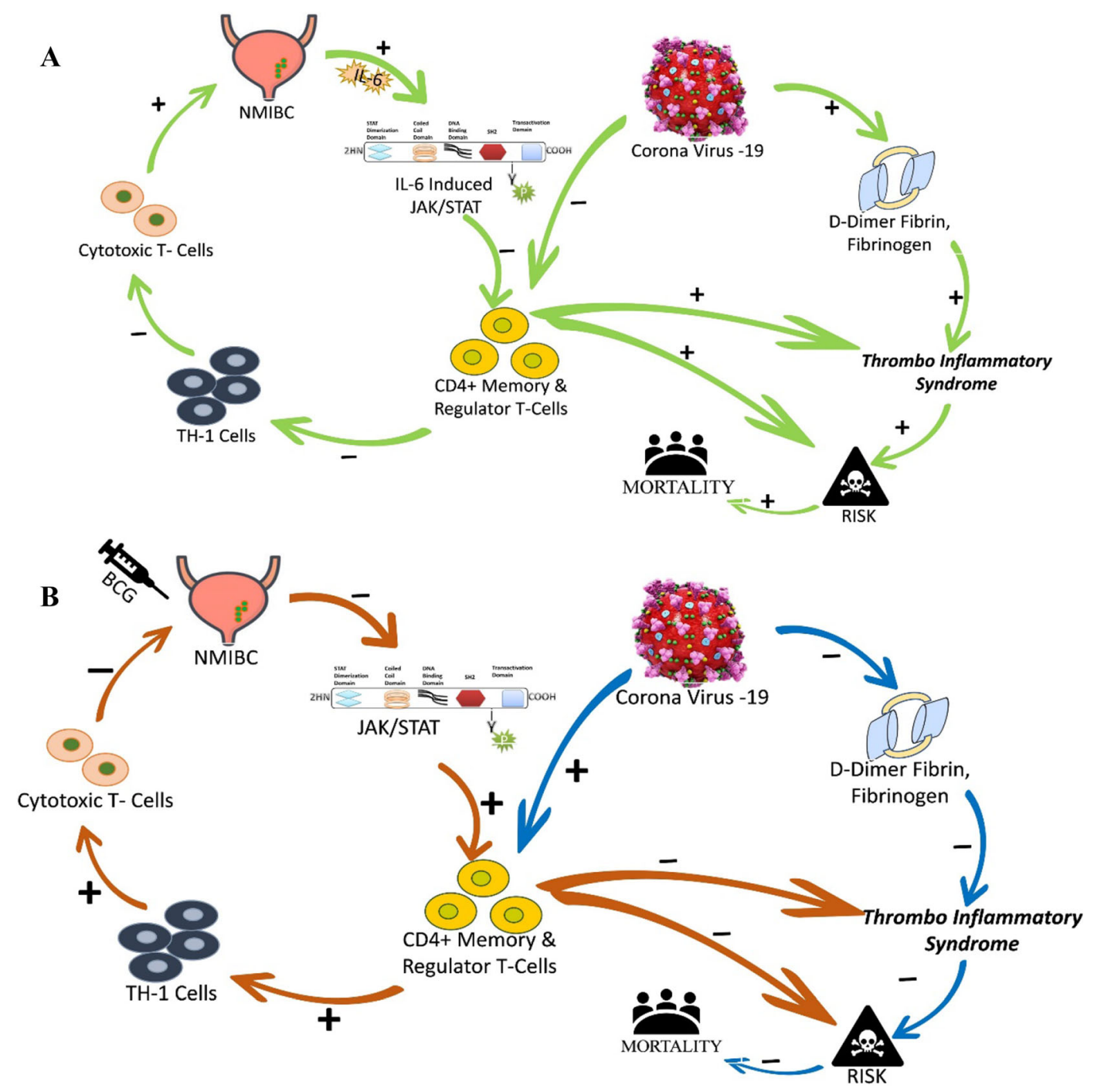

Fig. 1 a Canonical Pathway: The NMIBC patients upregulate the IL-6 mediate JAK /STAT pathway followed by inhibition of $\mathrm{CD} 4+$ memory and regulatory $\mathrm{T}$ cells with increase the thromboinflammatory syndrome induced mortality in patients with Coexisting condition to COVID-19 response in infected patients. Whereas, IL-6 induced JAK/STAT in NMIBC inhibit cytotoxic T cell activation and elevate the rate of proliferation and progression of NMIBC to high-risk category. b BCG induced Pathway: The BCG

the prevailing factor for two diseases associated with poor prognosis of NMIBC [3]. Dysregulated immune system with elevated rate of inflammation is therefore required to control and eliminate NMIBC and Covd-19. Similarly, viral-mediated inflammatory response causes extravasation of the neutrophil and plasma leakage within the affected tissue. Consequently, CD4 + T-cells, including effector, memory, and regulatory T-cells, are primarily affected by lymphopenia and reduced IFN- $\gamma$ expression in CD4 + Tcells with increased production of IL-6 [4]. The scientific knowledge revealed IL- 6 plays a key role in the progression and metastasis of cancer by regulating the cancer stem instillation in NMIBC patients downregulate the IL-6 mediated JAK/ STAT pathway with upregulation of CD4 + memory and receptor T cells with upregulation of cytotoxic T-Cells and affect the proliferation and progression of NMIBC. Furthermore, we proposed BCG induction in NMIBC patients with COVID-19 reduce the risk of mortality by alleveating the thrombi-inflammatory syndrome and could be a potential treatment for the COVID-19 infection with reduced mortality rate

cells and tumor microenvironment. The IL-6 mediated Janus kinase (JAK)/STAT3 pathway is hyperactive for many cancers, including NMIBC. The IL-6 mediated JAK/ STAT3 axis acts in the tumor microenvironment to supress the antitumor immune response and, on the other hand, to encourage tumor cell proliferation, survival, invasiveness, and metastases (Fig. 1a). IL-6 directly acts on tumor cells to induce STAT3 target gene expression, which encodes proteins that drive tumor proliferation and survival [5]. As a result, NMIBC patients exhibit exacerbated disease condition and higher rate of cancer mortality. However, the treatment and clinical management of NMIBC consistent 
with BCG-mediated intravesical adjuvant therapy. The heterologous protective function of BCG against antitumor and respiratory disease is considered. Previous studies have confirmed that BCG vaccination in neonates reduces the respiratory-driven mortality [6]. Current knowledge of BCG vaccination exhibits its mechanism and is known to be a non-specific protective role against respiratory infections. Subsequent pathways adopt heterologous immunity, which is influenced by antigen-independent activation of memory B and T cells. However, histone modification in the promoter region of the encoding gene for inflammatory cytokines in monocytes leads to a long-term immune response when reactivated (7). This defence includes trained immunity with a shielding effect in NMIBC against tumor progression and proliferation with the BCG instillation in patients (Fig. 1b). This non-specific effect of BCG is partially mediated by innate immune memory due to epigenetic changes in innate and adaptive immune system cells induced by microbe. However, Covid-19 induces dysregulation of immune system associated with adaptive and innate response, thus NMIBC have lower mortality and incidence among the common cancer types. Additionally, the need for novel and efficient therapeutic strategies for patients with severe Covid-19 will remain, since an effective vaccine or antiviral drugs against Covid-19 will developed. So, it is important to regularly test repurposed drugs includes anti-cancer drugs targeting immune dysfunction, coagulopathy and inflammation.

Author's Contribution Substantial contributions to the conception or design of the work and the acquisition; Drafting the work or revising it critically for important intellectual content.

\section{Declarations}

Conflicts of interest The authors declare that they have no conflict of interests.

\section{References}

1. Mehta V, Goel S, Kabarriti R, Cole D, Goldfinger M, AcunaVillaorduna A, et al. Case fatality rate of cancer patients with COVID-19 in a New York hospital system. Cancer discovery. 2020 Jul;10(7):935-41. PubMed PMID: 32357994. Pubmed Central PMCID: 7334098.

2. Dai M, Liu D, Liu M, Zhou F, Li G, Chen Z, et al. Patients with cancer appear more vulnerable to SARS-CoV-2: A Multicenter study during the COVID-19 outbreak. Cancer discovery. 2020 Jun;10(6):783-91. PubMed PMID: 32345594. Pubmed Central PMCID: 7309152.

3. Busetto GM, Porreca A, Del Giudice F, Maggi M, D'Agostino D, Romagnoli D, et al. SARS-CoV-2 infection and high-risk nonmuscle-invasive bladder cancer: are there any common features? urologia internationalis. 2020;104(7-8):510-22. PubMed PMID: 32516772. Pubmed Central PMCID: 7316644.

4. Chen Z, John Wherry E. T cell responses in patients with COVID19. Nature reviews Immunology. 2020 Sep;20(9):529-36. PubMed PMID: 32728222. Pubmed Central PMCID: 7389156.

5. Johnson DE, O'Keefe RA, Grandis JR. Targeting the IL-6/JAK/ STAT3 signalling axis in cancer. Nature Rev Clin Oncol. 2018 Apr;15(4):234-48. PubMed PMID: 29405201. Pubmed Central PMCID: 5858971.

6. Higgins JP, Soares-Weiser K, Lopez-Lopez JA, Kakourou A, Chaplin K, Christensen H, et al. Association of BCG, DTP, and measles containing vaccines with childhood mortality: systematic review. Bmj. 2016 Oct 13;355:i5170. PubMed PMID: 27737834. Pubmed Central PMCID: 5063034 www.icmje.org/coi_disclosure. pdf (available on request from the corresponding author) and declare: grant support from WHO for undertaking the research; no other support from any organisation for the submitted work; no other financial relationships with any other organisations that might have an interest in the submitted work in the previous three years; and no other relationships or activities that could appear to have influenced the submitted work.

7. van der Heijden C, Noz MP, Joosten LAB, Netea MG, Riksen NP, Keating ST. Epigenetics and trained immunity. Antioxidants and redox signaling. 2018 Oct 10;29(11):1023-40. PubMed PMID: 28978221. Pubmed Central PMCID: 6121175.

Publisher's Note Springer Nature remains neutral with regard to jurisdictional claims in published maps and institutional affiliations. 